toxicologique, Doin Paris (1924); Nrcloux, M., L'oxyde de carbone et l'intoxication oxycarbonéc, Masson Paris (1925). - 7. Kohn-Abrest, E., Précis de toxicologie, S. 37, Doin, Paris, (1955). - 8. Christmann, A. A. und E. L. Randall, J. biol. Chemistry 102, 595 (1933). - 9. Moureu, H., P. Chovin, L. Truffert und J. Lebbe, Arch. Maladies profess. Paris 2, 116 (1957). - 10. Ringold, A., P. Alto, J. R. Goldsmith, H. L. Helwig, R. FINN und F. SChUETTE, Arch. environm. Health 5, 308 (1962). - 11. Getrler, A. O. und H. C. Freimuth, Amer. J. Clin. Path. 13, 79 (1943). - 12. Seifert, P. und L. Schmieder, Dtsch. Zschr. gerichtl. Med. 41, 435 (1952). - 13. SACHs, V., DrägerHefte 235, 542 (1959). - 14. Gramer, L., Wschr. Unfallhk. 4, 121 (1961); Zbl. Arbeitsmed. Darmstadt 3, 53 (1961); Ärztl. Laborat. 12, 373 (1961). - 15. Massmann, W., Zschr. inn. Medizin 7, 293 (1956). - 16. Rovghton, F. J. W. und P. F. Scholander, J. biol. Chemistry 148, 551 (1943). - 17. Berninger, H. und R. Smith, Clin. Chem. N. Y. 5, 127 (1959). - 18. Franzen, E. und H. ThIEde, Z. ges. Hygiene Grenzgeb. 1, 44 (1964). - 19. FrETwURST, F. und F. Meinecke, Arch. Toxikol. 17, 273 (1959). - 20. Giubileo, M., Lavoro e med., Genova 49, 530 (1958). - 21. KuRz, H. und H. Dirkwaller, Arch. Toxikol. 15, 291 (1955). - 22. Zrjlstra, T. M., Klin. Wschr. 34, 384 (1956). - 23. WolfF, E., Nord. kriminaltekn. Tidskr. 11, 125 (1941); Ann. méd. lég. Paris 27, 221 (1947). - 24. Wennesland, R., Acta physiol. Scand. 1, 49 (1940). - 25. Allen, T. H. und W. S. Roor, J. biol. Chemistry 216, 319 (1955). - 26. Berka, J., Acta med. Scand. 152, 485 (1955). - 26 a. Fabre, R., R. Truhaut und F. Berrod, Ann. pharm. franr.. 9, 626 (1951). - 27. Feldstern, M. und N. C. KLendshoj, Chem. Abstr. 49, 410 b (1955). - 28. Le MOAN, G., Ann. pharmaceut, franç. 10,
269 (1952). - 29. Marquardr, W., Dtsch. Zschr. gerichtl. Med. 40, 385 (1951). - 30. MassmanN, W. und D. Sprecher, Arch. Gewerbepath. Berlin 14, 208 (1956). - 31. Nestorescu, B. und A. Roventa, Igiena 2, 165 (1959). - 32. Nitschke, M., Ann. méd. lég. Paris 33, 155 (1953). - 33. Truhaut, R. und C. Boudéne, Compt. Rend. Acad. Sci. France 256, 5453 (1963). - 34. Werst, H. J., Z. Ges. Hygiene 7, 504 (1961). - 35. Williams, L. A., R. A. LINN und B. ZaK, Amer. J. Clin. Path. 34, 334 (1960). - 36. Cruhandu, G. und V. Rusu, Z. analyt. Chem. 222, 393 (1966). - 37. Jones, J. G. und D. H. Walters, Ann. Occup. Hygiene 5, 221 (1962). - 38. Vignoli, L., Bl. Cristau, J.-P. Defretin und R. Vignoli, Arch. Mal. profess. Paris 21, 423 (1960). - 39. Ciunandu, G., Studii Cercetări Chimie 3/4, 189 (1956). - 40. FAcsko, G. und R. Minges, Talanta (London) 5, 102 (1960). - 41. Ciuhandu, G., D. Ceaușescu und G. Facsko, Studii Cercetări Chimie 1, 79 (1957). - 42. Cruhandu, G. und V. Gruran, Z. analyt. Chem. 159, 250 (1958). - 43. Crumandu, G., Studii Cercetări Chimie 3/4, 243 (1955). - 44. Ciuhandu, G., G. Krall und V. Giuran, Acta chim. Acad. Sci. hung. 28, 171 (1961). - 45. Ciuhandu, G. und G. Krall, Z. analyt. Chem. 172, 81 (1960). - 46. Ciuhandu, G., Studii Cercetări științ. 1/4, 133 (1955). - 47. Cruhandu, G., Z. analyt. Chem. 155, 321 (1957); 161, 345 (1958). - 48. Ciunandu: G. und G. KraII, Rev. Chimie 9, 531 (1959). - 49. Ciuhandu, G., V. Rusu und M. Diaconovicr, Z. analyt. Chem. 208, 81 (1965). 50. Levaggr, D. A. und M. Feldsten, Amer. ind. Hyg. Assoc. Quart. 25, 64 (1964). - 51. Cruhandu, G. und V. Rusu, Proceedings XV. Internat. Congr. Occup. Health, Wien 1966, S. 307. 52. Feldstein, M., J. forensic. Sci. 10, 1, 35 (1965).

Dr. G. Ciuhandu Str. Michelangelo 1 Timișoara, Rumänien

\title{
Dünnschichtchromatographische Trennung und halb-quantitative Bestimmung des Blut-Phenylalanins als Verfahren zur systematischen Prüfung auf Phenylketonurie bei Kleinstkindern
}

\author{
Von E. NITSChKE \\ Aus dem Institut d'Hygiène et de Santé Publique, Luxemburg (Direktor: Dr. F. Scbwacbtgen)
}

(Eingegangen am 4. Oktober 1967)

Es wird eine dünnschichtchromatographische Methode zur scharfen Trennung und zur halb-quantitativen Bestimmung des Plasmaphenylalanins angegeben. Der Phenylalanin-Fleck befindet sich, klar getrennt, über denjenigen der anderen Aminosäuren. Zur Verwendung gelangen Merck Fertigplatten Silicagel $\mathrm{F}_{254}$. Eine neue, spezifische Fluoreszenzmethode gestattet die einwandfreie Bestätigung der Befunde, so daß falsch-positive Resultate ausgeschlossen sind.

Wegen ihrer sicheren Aussage, ihrer Einfachheit und Billigkeit wird sie in unserem Land als screening test für die eben anlaufende systematische Untersuchung auf Phenylketonurie bei Kleinstkindern benutzt.

A thin layer chromatographic method is reported for the clean separation and semi-quantitative determination of phenylalanine in blood plasma. Ready-prepared plates of silicagel $\mathrm{F}_{254}$ (Merck) were used. The phenylalanine spot is clearly separated from all the other amino acids, and its identity is confirmed by a new, specific fluorescence reaction, which excludes any false positive results.

Owing to its specificity, simplicity and cheapness, we now use this method in the routine phenylketonuria screening test on newly born infants, which has just started in this country.

Ursache der Phenylketonurie ist die Abwesenheit eines spezifischen Enzyms, der Phenylalanin-hydroxylase ${ }^{1}$ ) im Organismus, wodurch die normale Umwandlung des Phenylalanins in Tyrosin nicht stattfinden kann. Es ent-

1) Enzyrne: Phenylalaninhydroxylase = Phenylalanin-4-hydroxylase (EC 1.99.1.2); L-Aminosäureoxydase = L-Aminosäure: $\mathrm{O}_{2}$ Oxydoreduktase (desaminierend) (EC 1.4.3.2). steht eine starke Anbäufung von Phenylalanin bis auf das 10-20fache der Normalwerte und gleichzeitig über einen Stoffwechselseitenweg eine starke Vermehrung der Phenylbrenztraubensäure, der Phenylmilchsäure, der Phenylessigsäure u. a. $\mathrm{m}$. Die Phenylbrenztraubensäure kann sehr leicht im Harn durch Eisenchlorid nachgewiesen werden (Phenistix-Teststäbchen). Diese Re- 
aktion ist jedoch nicht spezifisch und für eine Frühdiagnose nicht geeignet, da die Phenylketone erst etwa 6 Wochen post partum, d. h. zu einem Zeitpunkt, zu dem sich die Säuglinge nicht mehr in den Entbindungsanstalten befinden und einer systematischen Kontrolle nicht mehr zugänglich sind, in nachweisbaren Mengen im Harn erscheinen. Da dieser metabolische Fehler mit seinen verhängnisvollen irreversiblen Auswirkungen auf den Intelligenz-Quotienten durch eine geeignete, frühzeitig verabreichte phenylalaninarme Diät wirkungsvoll bekämpft werden kann, ist es notwendig, den Phenylalaningehalt des Säuglingsplasmas einige Tage nach der Geburt zu bestimmen (1).

Als geeignete Untersuchungsverfahren sind zu nennen: Die bakteriologische Methode nach Guthrie (2), die jedoch nur in bakteriologischen Instituten ausgeführt werden kann. Sie ist ziemlich ungenau, gibt relativ viele falsch-positive Resultate und ist auch nicht streng spezifisch, da Prolin ebenfalls die Hemmung des Wachstums durch $\beta$-2-Thienylalanin aufhebt.

Die en $y^{\prime \prime m a t i s c h e ~ M e t h o d e ~ m i t ~ L-A m i n o s a ̈ u r e o x y d a s e ~}$ nach LA Du und Mitarbeiter. Sie ergibt genaue Resultate, gestattet die Bestimmung von Tyrosin neben Phenylalanin, benötigt jedoch verhältnismäßig viel Blut und ist ziemlich teuer (3).

Die fluorometrische Methode (4) nach McCAMAN und RoBINs hat augenblicklich die größte Verbreitung. Sie bedingt jedoch die Anwesenheit eines teuren Fluoreszenz-Spektrophotometers und hat den Nachteil der relativen Unsicherheit von fluorometrischen Analysen. Es werden $50 \mu l$ Serum (etwa $150 \mu l$ Vollblut) benötigt.

Daneben werden auch verscbiedene papiercbromatographische Methoden vorgeschlagen. Grundsätzlich wären derartige chromatographische Verfahren vorzuziehen, da sie einfach auszuführen und viel billiger sind, sich gut $z u$ Serienuntersuchungen eignen und außerdem den nicht zu verkennenden Vorteil haben, neben einer Erhöhung des Phenylalanins auch Anomalien anderēr Aminosäuren anzeigen zu können.

So konnten wir in einem Falle mit unserer dünnschichtchromatographischen Methode beweisen, dạß ein nach McCAMAN gefundener erhöhter Phenylalaningehalt $(10 \mathrm{mg} / 100 \mathrm{~m} /)$, nicht durch einen spezifischen Enzymmangel bedingt war, da praktisch alle Aminosäuren gleichwertig vermehrț waren.

Die sehr einfache Methode nach Culley und Mertz (5) ergab bei uns keine befriedigenden Resultate. Auch die etwas aufwendigere Methode nach SCRIver (6) konnte nicht befriedigen, da sie nicht sehr empfindlich und zudem ziemlich ungenau ist. In Anlehnung an das Verfahren von GuthrIE geht man von Blutflecken aus, die autoklaviert und danach mit verdünntem Alkohol ausgezogen werden.

Es scheint verwunderlich, daß unseres Wissens bis jetzt in der Literatur keine dünnschichtchromatographische Methode erschienen ist, da doch die Dünnschichtchromatographie vor der Papierchromatographie viele Vorteile besitzt und auf dem Gebiet der Aminosäuren dieselbe immer mehr verdrängt.
Von einem derartigen Untersuchungsverfahren ist $\mathrm{zu}$ fordern:

1. Das Phenylalanin muß durch eine eindimensionale Entwicklung klar von den anderen Aminosäuren getrennt werden.

2. Neben der Identifizierung durch den $R_{\mathrm{F}}$ muß eine für Phenylalanin spezifische Nachweismethode zur Anwendung kommen.

3. Es wäre zwecks Erleichterung der Auffindung der betreffenden Flecke wünschenswert, daß dieselben entweder oberhalb oder unterhalb der anderen Anunosäuren liegen, $d$. h. also entweder einen höheren oder einen niedrigeren $R_{F}$-Wert haben.

Durch Hochspannungs-Dünnschichtelektrophorese (Arbeitsbedingungen: $700 \mathrm{~V}, 40 \mathrm{Min} .$, 10proz. Ameisensäure) konnten wir für das Phenylalanin eine geringere Wanderungsgeschwindigkeit gegenüber den anderen Aminosäuren erreichen (mit Ausnahme des Taurins, das praktisch am Startpunkt bleibt und somit nicht stört). In der Zwischenzeit konnten wir jedoch ein Fließmittel finden, in welchem das Phenylalanin einen deutlich höheren $R_{\mathrm{F}}$-Wert hat als die anderen, normal vorkommenden Aminosäuren. Es hat folgende Zusammensetzung: tert. Butanol/Äthylmethylketon/25proz. Ammoniak/Wasser : 30/30/10/10 (v/v).

Es gelang uns weiterhin, ein für Phenylalanin spezifisches Fluoreszenz-Nachweisverfahren auszuarbeiten. Besprüht man die Platte mit einem alkalischen Ninhydrinreagenz und nach der Farbentwicklung mit Natronlauge, so entsteht nach Erhitzen eine rein wreiße Fluoreszenz, welche von keiner anderen von uns geprüften Aminosäure gegeben wird. In Verbindung mit dem sehr günstigen $R_{\mathrm{F}}$-Wert liegt demnach eine absolut sichere Identifizierung des Phenylalanins vor, so daß falsch-positive Resultate im Gegensatz zu anderen Methoden ausgeschlossen sind. Nach der Testfleckenvergleichsmethode können die Phenylalaningehalte mit einer Genauigkeit von wenigstens $1 \mathrm{mg} / 100 \mathrm{ml}$ abgeschätzt werden.

\section{Methodik}

\section{Vorbereitung der Probe}

Zur Blutentnahme verwenden wir die heparinierten kalibrierten Kapillarröhrchen zur Mikro-Hämatokritbestimmung, die wie üblich verschlossen und in die mit laufenden Nummern versehenen Halter eingesteckt werden (Seal-ease holder der Firma ClayAdams).

Im Laboratorium werden sie in der Spezial-Mikrozentrifuge ausgeschleudert, in der Mitte zerbrochen und $25 \mu /$ des klaren Plasmas in Mikrozentrifugengefäße (Eppendorf) eingeblasen, in welche vorher $100 \mu l$ Äthanol p. a. eingefüllt wurden.

\section{Chromatographie}

Nach dem Mischen und Zentrifugieren werden mittels einer Hamiltonspritze $25 \mu l$ der überstehenden klaren, eiweißfreien alkoholischen Lösung auf die Platte aufgetragen (Föhn). Es soll Sorge getragen werden, daß die Flecken einen gleichmäßigen Durchmesser haben, der $3 \mathrm{~mm}$ nicht übersteigen soll.

Wir verwenden Merck Silicagel-Fertigplatten $(20 \times 20 \mathrm{~cm})$. Sie haben vor den selbsthergestellten Platten den Vorzug ciner gleichmäßigen und festeren Kieselgelschicht, die durch die Metallspitze der Hamiltonspritze nicht zerstört wird. 
Als Testlösungen verwenden wir zwecks besserer Vergleichsmöglichkeit ein Mischserum dessen Phenylalaningehalt nach McCaMan und Robins bestimmt wurde. Man begeht jedoch keinen nennenswerten Fehler, wenn man für dieses Mischserum einen Phenylalaningehalt von rund $2 \mathrm{mg} / 100 \mathrm{~m} l$ annimmt.

Dieses Serum wird mit einer 1proz. Phenylalaninlösung so angereichert, daß man eine Vergleichsreihe folgender Konzentrationen erhält: $2,0 \mathrm{mg} / 100 \mathrm{ml}$ (ohne Zusatz) 4, 6, 8 und $10 \mathrm{mg} / 100 \mathrm{ml}$. Im Kühlschrank aufbewahrt halten sich diese Seren etwa 2 Wochen.

Auf jede Platte werden 3 Vergleichsseren z. B. 2,4 und $10 \mu \mathrm{g} /$ $100 \mathrm{~m} /$, sowie die zu untersuchenden enteiweißten Proben (Abstand $12 \mathrm{~mm}$ ) aufgetragen. Es können jeweils 10-12 Proben aufgebracht werden. Die Entwicklung erfolgt aufsteigend bei Kammersättigung durchlaufend während $6 \mathrm{Stdn}$. $\mathrm{Zu}$ diesem Zweck wird vermittels Papierklemmen ein mehrfach gefaltetes Stück „Kleenex“-Papier an der oberen Kante der Platte befestigt, das sich mit dem übersteigenden Laufmittel vollsaugt. Laufmittel: tert. Butanol/Äthylmethylketon/25proz. $\mathrm{NH}_{3} /$ Wasser : 30/30/10/10 (v/v). Anschließend werden die Platten durch intensive Belüftung getrocknet und von Ammoniakspuren befreit. Hierzu eignen sich bestens die kleinen Heim-Heizlüfter. Die Trocknung soll bei $1000 \mathrm{~W}$, erste Stufe des Ventilators, wenigstens eine Stunde dauern.

\section{Naclbeis}

Auf die abgekühlte Platte werden $30 \mathrm{~m} l$ Ninhydrinlösung aufgesprüht (oder Tauchlösung). Diese Lösung hat folgende Zusammensetzung: $100 \mathrm{mg}$ Ninhydrin in $30 \mathrm{~m} l$ Aceton gelöst, plus $2 \mathrm{ml}$ Triäthylamin. Sie soll jedesmal frisch hergestellt werden. Die Farbentwicklung erfolgt ebenfalls unter dem Heizlüfter bis zur maximalen Farbintensität (15-30 Min.). Die Phenylalaninflecke befinden sich mit klarem Abstand über den anderen Aminosäureflecken. Bei heparinierten Proben erscheint ein ninhydrin-positiver Fleck, welcher mit größerem Abstand über den PhenylalaninFlecken liegt und somit nicht stört. Sie haben eine bräunlich-blaue Farbe, die bei normalen Gehalten zwar schwach, jedoch deutlich erkennbar ist. Wegen der günstigen Lage der Phenylalaninflecken ist auch bei ungleichmäßiger Entwicklung eine Verwechslungsgefahr nicht gegeben.

In den seltenen Fällen, wo eine Probe einen gegenüber der Vergleichslösung von $2 \mathrm{mg} / 100 \mathrm{~m} /$ intensiv gefärbten Phenylalaninfleck aufweist, wird das spezifische Nachweisverfahren angewendet. Auf die waagerechte Platte wird $\mathrm{N}$ Natronlauge aufgesprüht bis zur völligen Durchnässung der Kieselgelschicht. (Sie soll jedoch nicht „schwimmen“). Es ist nicht nötig, die ganze Platte zu besprühen. Die Platte wird sofort waagerecht in einen auf $90^{\circ}$ gebrachten Heizschrank gelegt und hier wenigstens eine halbe Stunde belassen. Nach dem Erkalten ergeben die Phenylalaninflecken unter der langwelligen Analysenquarzlampe eine spezifische weiße Fluoreszenz. Die Empfindlichkeit hängt naturgemäß von der verwendeten Quarzlampe ab. Unter unserer Quarzlampe älteren Modells ist die Fluoreszenz der normalen Phenylalaningehalte eben zu erkennen, während erhöhte Werte eine stärkere Fluoreszenz ergeben, die der Konzentration proportional ist.

\section{Quantitative Bestimmung}

In den Ausnahmefällen, wo ein erhöhter Phenylalaningehalt festgestellt wurde, wird eine neue Platte angesetzt mit den Vergleichslösungen von $2,4,6$ und $8 \mathrm{mg} / 100 \mathrm{ml}$, sowie je nach dem geschätzten Gehalt der Probe 5, 10,15 oder $20 \mu l$ der alkolischen enteiweißten Probelösung. ( $\mathrm{Da}$ das ursprüngliche Volumen $125 \mu \mathrm{l}$ betrug, steht genügend Probelösung zur Verfügung; es ist also nicht nötig, eine neue Blutentnahme vorzunehmen.) Die Behandlung der Platte erfolgt wie vorher angegeben. Es kann ohne besondere Ubung eine Abschätzgenauigkeit von mindestens $1 \mu \mathrm{g} /$ $100 \mathrm{ml}$ erreicht werden.

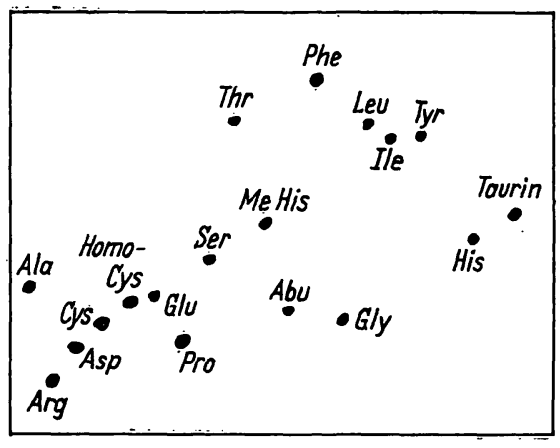

Abb. 1

Trennung von 18 Aminosäuren auf Merck-Fertigplatten Silicagel $20 \times 20 \mathrm{~cm}$.

Fließmittel : tert. Butanol/Äthylmethylketon/25proz Ammoniak/. Wasser: 30/30/10/10 $(\mathrm{v} / \mathrm{v})$, aufsteigend. Laufstrecke $18 \mathrm{~cm}$

Wilie oben gesagt besteht die Möglichkeit, im selben Arbeitsgang Anomalien der anderen Aminosäuren zu erkennen. Die eindimensionale Chromatographie erlaübt es allerdings nicht, Aussagen über alle Aminosüuren zu machen, da eine Trennung aller Aminosäuren niicht erreicht werden kann. Wie aus unserer Abbildung eisichtlich, ist Tyrosin nicht von Leucin, Isoleucin und Threonin getrennt. Eine starke Erhöhung des Tyrosingehaltes kann an einer intensiven bräunlich-blauen Farbe des unterhalb des Phenylalaninfleckens liegenden Flecks erkannt werden. Die Identifizierung muß jedoch getrennt vorgenommen werden mit $\alpha$-Nitroso- $\beta$-NaphtholReagenz nach Acher (7). Wir glauben jedoch, daß es nötig ist, in allen anomalen Fällen den Nachweis und die Abschätzung durch eine zweidimensionale Chromatographie vorzunehmen, sowie dies ja auch von Brckel gefordert wird (1).

\section{Literatur}

1. Bickel, H. und J. Bremer, Dtsch. med. Wschr. 92, 691 (1967). - 2. Guthrie, R. und A. Susi, Pediatrics Springfield 32, 338 (1963). - 3. LA Du, B. N., Pediatrics Springfield 31, 39 (1963). 4. McCaman, M. W. und E. Robins, J. Laborat. Clin. Med. (S.
Louis) 59, 885 (1962). - 5. Culley, W. J., J. Mértz und Mitarbeiter, Clin. Chem. New York 8, 266 (1962). - 6. SCriver, C. R., Lancet II 231 (1964). - 7. ACHER, R. und C. Crocker, Biochim. biophysica Acta (Amsterdam) 9, 704 (1952).

E. Nitschké

Luxembourg, 42, rue du Laboratoire 